\title{
SPRACHWISSENSCHAFT
}

http://dx.doi.org/10.18778/2196-8403.2007.11

\author{
WALDEMAR CZACHUR
}

\section{Konfrontative Textlinguistik am Beispiel deutscher und polnischer Vereinssatzungen}

\begin{abstract}
Celem artykułu jest dyskusja nad niektórymi aspektami porównania statutu jako rodzaju tekstu w języku polskim i niemieckim. Omówione zostaną podstawowe pojęcia lingwistyki tekstu i opracowane zadania konfrontatywnej lingwistyki tekstu, które tworzą podstawę do analizy polskich i niemieckich statutów. Badana jest funkcja statutów w kontekście ich użycia jak i ich struktura oraz środki językowe, które zostały zastosowane do wyrażenia normatywności w statutach.

Das Ziel des vorliegenden Beitrags ist es, einige Aspekte des Vergleichs der Textmuster Satzung im Deutschen und Polnischen zu diskutieren. Nach der Darstellung der grundlegenden Begriffe der Textlinguistik werden Aufgaben der konfrontativen Textlinguistik ausgearbeitet, auf deren Grundlage die Analyse der Vereinssatzungen im Deutschen und Polnischen erfolgt. Untersucht wird die Funktion der Textsorte Satzung im Verwendungskontext, insbesondere ihre Struktur und die sprachlichen Mittel, die die Normativität in den Satzungen beider Sprachen ausdrücken.

The aim of the present paper is a comparative discussion of text forms of association statutes in German and Polish. First the author reviews the constitutional concept of text linguistics and elaborates on the role of confrontational text linguistics. This is the basis for an analysis of statute texts in German and Polish. The function of the statute will be explored in its context, its structure and with regard to its linguistic means, which express the normativity of the statute.
\end{abstract}

\section{Vorbemerkungen}

Die Text(sorten)linguistik hat in den letzten Jahren sehr viele und zugleich unterschiedliche Ansätze zur Klassifikation und Beschreibung mannigfacher Textsorten entwickelt. Forschungsprojekte zur kontrastiven Textsortenlingui- 
stik sind jedoch noch rar. Die ersten Versuche, deutsch-polnisch kontrastiv orientierte textlinguistische Untersuchungen vorzunehmen, haben WAWRZYNIAK $(1975,1989)$ und GRUCZA (1993) unternommen. Während sich Wawrzyniak eher für die Anwendung der Erkenntnisse der konfrontativen Textlinguistik interessiert und nach Textbildungsregularitäten in beiden Sprachen sucht, plädiert Grucza für die Übernahme eines dynamischen Textmodells für die konfrontative Analyse. Die in den letzten Jahren in Polen entstandenen Arbeiten zur kontrastiven Textlinguistik beschäftigen sich mit einigen Aspekten des textlinguistischen Vergleichs (HeINEMANN / BILUT-HoMPLEWICZ 2005), jedoch sind bisher keine theoretischen Grundfragen für eine deutsch-polnische kontrastive Text(sorten)linguistik erörtert worden (ADAMZIK 2001). Eine Ausnahme bildet die Arbeit von BILUT-HomPLEWICZ (2004), in der zehn Anforderungen an die deutsch-polnische und polnisch-deutsche kontrastive Linguistik im Hinblick auf die Textsortenforschung aufgelistet werden. Vor diesem Hintergrund soll versucht werden, die Vereinssatzung als Textsorte im Polnischen und Deutschen gegenüberzustellen und auf die Form, Inhalt und Struktur dieser Textsorte in beiden Sprachen aufmerksam zu machen. Dabei soll es vor allem darum gehen, den Grad der Standardisierung und ihre Ursachen sowie die sprachlichen Mittel zur Realisierung einzelner Sprechakte zu vergleichen.

\section{Zum Konzept Textsorte und Textmuster}

Vergleicht man zwei Sprachen in textlinguistischer Hinsicht miteinander, sollten zunächst die zu vergleichenden Einheiten definiert werden. Der Ansatz, dass sich die Textlinguistik mit Texten beschäftigt und folgerichtig bei einer konfrontativen Untersuchung zwei Texte gegenübergestellt werden sollten, ist aus pragmatischer und kognitiver Sicht nicht ausreichend und wissenschaftlich wenig ergiebig. Die Textlinguistik bedient sich daher einer Kategorie, die einen Text auf einer abstrahierten Ebene repräsentiert und eine Beschreibung, eine Klassifikation sowie einen Vergleich ermöglicht. Sowohl im Deutschen als auch im Polnischen gibt es eine große und zugleich verwirrende Vielfalt von Termini zur Bezeichnung ein und derselben Kategorie. ${ }^{1}$ Gegenwärtig wer-

1 Im Deutschen sind folgende Begriffe zu finden: ,Texttyp (GÜLICH/RAIBLE 1975; WERLICH 1975; IsENBERG 1978), ,Textform “ (WERLICH 1975), ,Textmuster" (SANDig 1986, 1997; Michel 1990; LeRChNer 1991; HeinEMANn 2000), , Textklasse ' (HARTMANN 1971; DiMTER 1981; STEGER 1983), ,Textart‘ (MISTRIK 1973) und ,Diskurstyp‘ (S. J. SchмiDT 1976). In der polnischen Sprachwissenschaft werden u.a. folgende Begriffe verwendet: ,gatunek', ,gatunek mowy‘ (BARTMIŃSKI 1998), ,model gatunku / tekstu‘, ,wzorzec gatunkowy“ (GAJDA 1993; WiTOSZ 
den in der Textlinguistik vorwiegend zwei Begriffe verwendet: Textsorte und Textmuster. Während Textmuster als „Klassen geistiger Erfahrung“ (HöRMANN 1977:172) definiert werden, die aus Abstraktionsprozessen als ein bewährtes und zugleich ideales Orientierungsmuster für die Bewältigung spezifischer kommunikativer Aufgaben entstehen, sind Textsorten dagegen für HEINEMANN / HEINEMANN (2002) etwas Konkretes, also die Repräsentationsformen eines Textmusters auf niederer Abstraktionsstufe, die auch atypische Merkmale aufweisen. Die Textmuster bilden sich allmählich auf Grund individueller kommunikativer Erfahrungen und gesellschaftlicher Lernprozesse in einem kulturellen und damit historisch definierten Raum aus. Sie sind also kognitive Vorgaben für die Produktion und Rezeption von Texten, die sich durch Wesensmerkmale wie Repetitivität, Vagheit, Flexibilität und Variabilität kennzeichnen lassen. Die praktische Umsetzung dieser kommunikativen Muster erfolgt in Form von Textexemplaren. Sie werden dann weiter zwecks der besseren Identifizierbarkeit und Verwendung virtueller Textklassen auf niederer Abstraktionsstufe zugeordnet, d.h. den Textsorten. Die Textsorten sind somit merkmalhaltiger und lassen sich beschreiben ,als Sammelbegriff für eine finite Menge von - durch Übereinstimmung bestimmter textkonstitutiver Merkmale gekennzeichneten - virtuellen Textexemplaren“ (HEINEMANN 2000:518). Auch SANDIG (1997:26) definiert die Textmuster als „ein standardisiertes (konventionelles) Mittel zur Lösung in einer Gesellschaft auftretender Standardprobleme" und beschreibt sie weiter als Zusammenhang von (nicht sprachlichem) Handlungstyp und (sprachlicher) Textsorte. Demzufolge ist die Textsorte „das standardisierte Handlungsmittel, mit dem Handlungen nach dem Handlungstyp vollzogen werden können“ (SANDIG 1997:27). Das bedeutet, dass der Handlungstyp die konventionalisierte Erwartung bezüglich der Textsorte steuert und die Textsorteneigenschaften den Handlungstyp „kontextualisieren“, der konventionell mit ihnen verknüpft ist. In Anlehnung an HEINEMANN / HEINEMANN (2002:138) sei hier bemerkt, dass Textmuster als kognitive Operationen zur Konstitution und zum Verstehen von Texten zu betrachten sind, die sich einem bestimmten Textsortenrahmen zuordnen lassen, wobei hier keine 1:1-Entsprechung anzunehmen ist. Wie RIEHL (2001:96) in diesem Zusammenhang zu Recht anmerkt, ,[hat] jede Textsorte [...] ein konventionalisiertes Textmuster, aber nicht jedes Textmuster ist automatisch auf eine Textsorte bezogen". Hinzu kommt der Faktor, dass die Teilnehmer einer Sprachgesellschaft imstande sind, bestimmte kommunikative Aufgaben unter

2001; WoJTAK 1999), ,wzorzec tekstowy ' (ŻMIGRODZKA 1997; WyrWAS 2002), ,schemat tekstu“ (BONIECKA 1999) oder auch ,prototyp gatunku‘ (WITOsZ 2001). 
unterschiedlichen Situationsbedingungen zu bewältigen, da sie auf bestimmte als erfolgreich identifizierte Muster zurückgreifen können und zugleich fähig sind, die konkreten Texte bestimmten Textklassen, also den Textsorten zuzuordnen. Textsorten werden hier als Repräsentation des ihnen zugrunde liegenden Textmusters aufgefasst. ADAMZIK (2001:28), die auf diese Dichotomie verzichtet und gerne den Begriff ,Textsorte“ als „,Routineformel auf der Textebene“ verwendet, weist jedoch auf den wichtigen Aspekt ,,der historisch gewachsenen Einheiten der kommunikativen Praxis einer Gesellschaft" hin. Routine als „eine erfahrungsabhängige und automatisierte Fertigkeit“ (WARNKE 2001:242) ist ebenso konventionalisiert und dadurch kulturabhängig. Deswegen sollte bei der Diskussion um das Textmuster und die Textsorte mitberücksichtigt werden, dass durch die Aneignung dieser Einheiten die Annahme der kulturspezifischen Welterfassung, also bestimmter Formen zur Kategorisierung und Verarbeitung der Welt erfolgt. Diese Weltaneignung bildet zugleich die Grundlage des Textmusterwissens als kulturelles Wissen. WARNKE (2001:248) weist zu Recht darauf hin, dass die Textmuster nicht nur Teilaspekte einer Kultur sind, sondern auch Bausteine derselben, da sie Aspekte der Kultur generieren. Aus diesen Prämissen ergeben sich für die deutsch-polnische konfrontative Textlinguistik folgende Forderungen:

- Die konfrontative Untersuchung der Textmuster sollte zunächst den Verwendungskontext in beiden sprachlichen Kulturgemeinschaften analysieren.

- Dem Vergleich der beiden Textmuster sollte der gesellschaftliche Zweck sowie dessen Plan/Mittel zugrunde liegen.

- Die konfrontative Analyse sollte sich daher auf die einzelnen Teilhandlungen der zu vergleichenden Textmuster sowie auf ihre sprachliche Realisierung konzentrieren.

Diese eher allgemein formulierten Postulate sollen am Beispiel deutscher und polnischer Vereinssatzungen veranschaulicht werden.

\section{Das Vereinswesen in Deutschland und in Polen}

Bevor das Textmuster Satzung in beiden Sprachen verglichen wird, ist es wichtig, zunächst den Geltungsbereich dieser Textsorte in beiden Kulturräumen gegenüberzustellen, um die Unterschiede und Gemeinsamkeiten im Vereinswesen (geschichtlicher, rechtlicher und gesellschaftlicher Natur) aufzuzeigen. Das Vereinswesen ist in Deutschland und in Polen fester Bestandteil der Gesellschaft, auch wenn sich seine Entwicklungswege in den letzten zwei 
Jahrhunderten unterschiedlich gestaltet haben. Beide Länder haben eine auf Traditionen und u. a. auf christlichen Werten basierende Geschichte des Vereinswesens, insbesondere im sozialen Bereich, wie der Unterstützung von Hilfsbedürftigen und Armen. Der Verein als ein freiwilliger, auf Dauer angelegter, sich selbst verwaltender Zusammenschluss von natürlichen oder juristischen Personen, der selbständig seine Ziele und Tätigkeitsbereiche bestimmt, einen gemeinsamen Namen trägt, sich von hierzu bestimmten Mitgliedern vertreten lassen kann und in dem jeder im Rahmen der Satzung nach freien Stücken ein- und austreten kann, ist bedeutender Bestandteil der demokratischen Gesellschaft. Die (eingetragenen) Vereine sind rechtsfähig, das heißt, sie können als Rechtssubjekte selbst Träger von Rechten und Pflichten sein, sie sind auch strukturell unabhängig vom Staat, meistens auch nicht gewinnorientiert. Sie haben eine selbstverwaltende Organisationsform und eine konkrete soziale Funktion. Die Funktion des Vereinswesens in einer Gesellschaft bezieht sich zum einen auf die Ziele jedes Vereins, der in einem bestimmten Bereich tätig ist, und zum anderen auf dessen gesellschaftlich-soziale Mission. Dazu gehören nach LEŚ (2000:232) die Realisierung von materiellen und nichtmateriellen sozialen Dienstleistungen, die Ausgleichstätigkeit hinsichtlich des Gleichgewichts zwischen den Bürgerrechten und den sozialen Pflichten und die Werbung für Solidarität als Heilmittel für gesellschaftliche Krisen. Extremem Individualismus soll entgegengewirkt werden, und eine auf der Wohlfahrtsidee basierende Gesellschaftsordnung, die das Verantwortungsgefühl des Individuums, der Gemeinschaft und des Staates stärkt, soll gefördert werden. Dazu gehört die Kontrolle der öffentlichen Verwaltung und des privaten Sektors sowie die Bildung neuer Modelle für die gesellschaftliche Partizipation. Die rasche Entwicklung des Vereinswesens in beiden Ländern in den letzten zehn Jahren ist auf die Krise des Wohlfahrtstaates sowie auf die tiefgreifenden Wandlungsprozesse (neue politische Ordnung in Europa, Globalisierung) zurückzuführen. Da Vereine als typische (sich selbstverwaltende) Organisationen bürgerlichen Lebens gelten, werden sie auch Träger der Zivilgesellschaft genannt. Somit bildet die Zivilgesellschaft eine Gesellschaft aktiver Bürger, die durch politische Partizipation und Mitbestimmung auch und gerade jenseits der formalen politischen Institutionen das Gemeinwesen eines Staates gestalten (SACHSSE 2002:3). Darüber hinaus werden die Vereine auch als Orte der politischen Schule für Bürger bezeichnet. Betrachtet man das gegenwärtige deutsche Vereinswesen, so fällt zunächst auf, dass die Vereinskultur ein etablierter Bestandteil der Gesellschaft ist, der auch tief in der Mentalität verankert ist. Sie basiert auf dem traditionsreichen Prinzip der Selbstverwaltung und der Subsidiarität sowie auf der Idee der sozialen Solidarität 
(Preußische Städteordnung vom November 1808). Auch die rechtlichen Regelungen haben eine lange Geschichte (Allgemeines Landrecht für Preußische Staaten von 1794, Bürgerliches Gesetzbuch von 1900). Die Vereine in Polen $^{2}$ haben ihre Tätigkeit hingegen erst nach 1989 reaktivieren können und wurden schnell zu einem Instrument der Demokratisierung der gesellschaftlichen Sphäre. LEŚ (2000) schreibt dem polnischen Vereinswesen neoliberaletatische Merkmale zu, da die polnischen Vereine eine politisch-gesellschaftliche Vermittlungsinstanz darstellen und eher die Funktion eines Kunden als die eines Partners für den öffentlichen Sektor erfüllen. Im Vergleich zu Deutschland ist das Vereinswesen und das damit verbundene bürgerliche Engagement in Polen mit Vorbehalten belastet und im weiteren Sinne auch nicht allgegenwärtig. In diesem Zusammenhang stellt sich die Frage, inwieweit sich die unterschiedlich funktionierenden Geltungsbereiche der Textsorte Satzung auf der textuellen und sprachlichen Ebene widerspiegeln. Dabei ist zu prüfen, wie die Vereinssatzungen (als für die Vereine grundlegenden Texte) aufgebaut sind, wie sie die Vereinswirklichkeit kreieren und wo die Gemeinsamkeiten und Unterschiede im deutsch-polnischen Vergleich liegen. Es ist zu erwarten, dass die unterschiedlichen Hintergründe des Vereinswesens in beiden Ländern, auch wenn sie sich in der Funktion überschneiden, einen unterschiedlichen Einfluss auf die sprachliche Realisierung der Textsorte Satzung haben.

\section{Handlungskontext und gesellschaftlicher Zweck}

Die rechtliche Grundlage der Vereinsbildung bildet die im deutschen Grundgesetz (Artikel 9, §1: „Alle Deutschen haben das Recht, Vereine und Gesellschaften zu bilden.“) und in der polnischen Verfassung (Artikel 58, § 1: ,Jedermann wird die Vereinigungsfreiheit gewährleistet.") garantierte Vereinigungsfreiheit. Es ist allen Vereinen gemeinsam, dass sie als freiwillige, auf eine gewisse Dauer angelegte, körperschaftlich organisierte Zusammenschlüsse einer Anzahl von Personen den Bürgern die Möglichkeit geben, sich aktiv am sozialen, kulturellen, wissenschaftlichen, wirtschaftlichen und sportlichen Leben einer Gesellschaft $\mathrm{zu}$ beteiligen und dadurch ein konkretes gesellschaftliches Ziel verfolgen zu können. Trotz der inhaltlichen Vielfalt ist die Organisationsform der Vereine gleich. Als gesellschaftliche Organe sind Ver-

2 Damit ist das ganze Vereinswesen in Polen gemeint, das sich in der Zwischenkriegszeit entwickelte, dann jedoch zu kommunistischen Zeiten einer strengen Kontrolle des Staates unterlag. Daher kann die Tätigkeit einiger polnischer oder internationaler Vereine bis zur Wende nicht als Instrument der Demokratisierung angesehen werden. 
eine bestimmten politischen, verwaltungstechnischen und wirtschaftlichen Verfahren und Mechanismen ausgesetzt, die ihre Handlungs- und Kommunikationsmuster prägen (CZACHUR 2005:112). Die Gründung und die Tätigkeit der Vereine sind durch entsprechende Gesetze in beiden Ländern geregelt. In Deutschland sind die Bestimmungen über das Vereinswesen im Bürgerlichen Gesetzbuch (BGB) ${ }^{3}$ verankert, in Polen regelt dies das Gesetz über die Vereine (Ustawa „Prawo o stowarzyszeniach“, UPS, aus dem Jahre 1989). Vor der Herausarbeitung weiterer Unterschiede zwischen dem deutschen und polnischen Vereinswesen sei jedoch kurz auf die Spezifik von Vereinssatzungen als normative Texte eingegangen. Die Vereinssatzungen bilden das normative Grundgerüst des Vereinslebens und haben eine rechtliche Leit- und Integrationsfunktion, indem sie die Grundwerte und Ziele des Vereins nennen, mit denen sich die Mitglieder identifizieren. Die Besonderheit der Vereinssatzungen besteht darin, ,[...] dass bisher nicht als Tatsachen geltende Sachverhalte bestimmter Art beschrieben werden, die durch den Vollzug einer Handlung dieses Typs Tatsachengeltung erhalten“ (VIEHWEGER / SPIES 1987: 89). Mit den Festlegungsakten, die eben in den Satzungen kodifiziert sind, werden Normen geschaffen, die das Vereinsleben bestimmen. Norm wird hier verstanden als eine Festlegung, die ,[...] einer Klasse von Handlungsträgern in wiederkehrenden Situationen und unter gleichbleibenden Bedingungen eine Klasse von Handlungen/Verhaltensweisen vorschreibt" (VIEHWEGER / SPIES 1987:94). Das größere soziale Gewicht, das diesen Normen zukommt, besteht darin,

- dass es langfristig wirkende Handlungsantriebe sind,

- dass mit ihnen soziale Gruppen konstituiert werden, definiert durch für sie geltende Rechte und Pflichten,

- dass diese Rechte und Pflichten das Verhalten der sozialen Gruppe in langfristigen und wiederholt stattfindenden komplexen Handlungsabläufen von gesellschaftlicher Bedeutung bestimmen,

3 Das BGB regelt als zentrale Kodifikation des deutschen allgemeinen Privatrechts die wichtigsten Rechtsbeziehungen zwischen Privatpersonen. Nach langjähriger Beratung in zwei Juristenkommissionen trat das BGB am 1. Januar 1900 durch Art. 1 des Einführungsgesetzes zum Bürgerlichen Gesetzbuch (EGBGB) in Kraft (RGB1. 195). Der Gesetzgeber hat seitdem sehr viele Änderungen am BGB vorgenommen. Es gilt in der Bundesrepublik Deutschland als Bundesrecht nach Art. 123, Abs. 1, Art. 125 GG fort. Am 2. Januar 2002 erfolgte eine Neubekanntmachung in neuer deutscher Rechtschreibung und mit amtlichen Paragraphenüberschriften. 
- dass die geschaffenen/festgelegten Rechte und Pflichten und die Handlungskontexte, in denen sie gelten, auf Systeme von gesamtgesellschaftlichen Werten und Zielen bezogen bzw. an ihnen orientiert werden. (VIEHWEGER / SPIES 1987:94)

Die obigen Punkte machen den gesellschaftlichen Zweck der Vereinssatzung deutlich, die aus zwei unterschiedlichen Perspektiven betrachtet werden soll. Zum einen ist die Satzung ein strukturelles und inhaltliches Grundgerüst für die Mitglieder, zum anderen stellt sie auch eine Grundlage zur Eintragung in das Vereinsregister dar (in Deutschland durch das örtliche Amtsgericht, in Polen durch das Krajowy Sąd Rejestrowy, KSR, geführt). Das bedeutet, dass die Verfasser einer Satzung zwischen der Nüchternheit der juristischen Sprache (Organisationsfunktion, eine rechtliche Leitfunktion) und dem Deklamatorischen (kollektive Integrationsfunktion) balancieren müssen.

\section{Plan/Mittel}

Der Aufbau von Vereinssatzungen wird in den gesetzlichen Bestimmungen über das Vereinswesen vorgeschrieben. Sowohl BGB ${ }^{4}$ als auch UPS enthalten Bestimmungen zu den für jede Satzung konstitutiven Elementen, die sich folgendermaßen darstellen lassen:

\begin{tabular}{|l|l|}
\hline Bürgerliches Gesetzbuch vom 2. Januar & $\begin{array}{l}\text { Ustawa „Prawo o Stowarzyszeniach“ } \\
\text { vom 7. April } 1989 \text { (Dz. U. Nr. 20, poz. 104) }\end{array}$ \\
\hline - Name und Sitz des Vereins, & • Name des Vereins, \\
- Zweck des Vereins, & - Wirksamkeitsgebiet und Sitz, \\
- Rechte und Pflichten der Mitglieder, & - Zweck und Realisierungsmittel, \\
- Regelung über den Eintritt und Aus- & - Regelung über den Erwerb und Verlust \\
tritt, & der Mitgliedschaft, Rechte und Pflich- \\
- Beitragsregelungen, & ten der Mitglieder, \\
- Vereinsorgane, ihre Bestellung und & - Vereinsorgane, ihre Bestellung und \\
Kompetenzen, & Kompetenzen, \\
& \\
\hline
\end{tabular}

4 Die dafür notwendigen Regelungen sind im BGB unter „Juristische Personen“, Untertitel „Vereine“ zu finden. Dieser Untertitel ist in zwei Kapitel gegliedert, im ersten Kapitel sind die „Allgemeinen Vorschriften“ (\$21-54) und im zweiten „Regelungen über den eingetragenen Verein“ (\$55-79) enthalten. Wichtig sind $\S 57$ „Mindesterfordernisse an die Vereinssatzung“ und $§ 58$,Sollinhalt der Vereinssatzung“. 


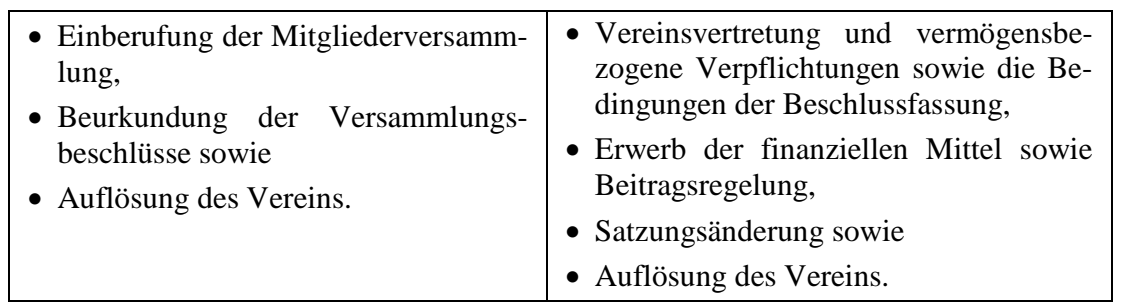

Vereinssatzungen müssen auf der Grundlage dieses Regelwerks verfasst werden, das auch Inhalt und Reihenfolge der Überschriften in hohem Maße festlegt. Vergleicht man die Vorgaben für die Produktion des Textmusters Satzung in beiden Rechtssprechungen, so fällt zunächst auf, dass die polnische Gesetzgebung diesbezüglich detailliertere Bestimmungen vorgibt, obgleich in den meisten Bereichen große Übereinstimmung festzustellen ist. Während das polnische Gesetz Regelungen bezüglich der Realisierungsmittel der Vereinsziele sowie des Vermögens vorschreibt, haben die deutschen Satzungen Regelungen über die Beurkundung der Versammlungsbeschlüsse zu enthalten. Dabei muss jedoch erwähnt werden, dass die obengenannten, als obligatorisch geltenden Satzungselemente jeweils noch um weitere vereinsspezifische Regelungen ergänzt werden können. In Anlehnung an MAZURs (1997) Differenzierung zwischen einem präskriptiven und einem deskriptiven Textmuster kann festgehalten werden, dass die oben dargestellte Struktur die Grundlage des präskriptiven Textmusters darstellt.

\section{Analyse}

Zur Beschreibung des Inhalts der jeweiligen Konstituenten und dementsprechend des deskriptiven Textmusters soll die von SCHRÖDER (2003) entwickelte ,indem-Methode" dienen. Es wird angenommen, dass sich die Texthandlung als komplexe Handlung beschreiben lässt, da sie eine Handlung ist und aus mehreren aufeinanderfolgenden und zusammenhängenden Teilhandlungen besteht. Daher wird der Zusammenhang zwischen den Teilhandlungen als ,indem-Zusammenhang' beschrieben: Eine Texthandlung wird vollzogen, indem eine Folge von Teilhandlungen vollzogen wird.

\begin{tabular}{|l|l|}
\hline Vereinssatzung nach deutschem Recht & Vereinssatzung nach polnischem Recht \\
\hline $\begin{array}{l}\text { Der Name und der Sitz wird definiert, } \\
\text { indem } \\
\begin{array}{l}\text { - der Name, der Sitz und die Rechts- } \\
\text { form festgelegt werden. }\end{array}\end{array}$ & $\begin{array}{l}\text { Die Allgemeinen Bestimmungen wer- } \\
\text { den geregelt, indem } \\
\text { ed Name, der Sitz sowie das Wirk- } \\
\text { samkeitsgebiet bestimmt werden. }\end{array}$ \\
\hline
\end{tabular}


Der Zweck des Vereins wird definiert, indem

- die strategischen und operativen Ziele aufgelistet werden,

- die konkreten Mittel der Zielrealisierung genannt werden.

Die Mitgliedschaft wird geregelt, indem

- die Bedingungen des Eintritts, Austritts und des Ausschlusses sowie die Rechte und Pflichten der Mitglieder festgelegt werden,

- die von den Mitgliedern zu leistenden Beiträge geregelt werden.

Die Organisationsstruktur des Vereins wird definiert, indem

- die Mitgliederversammlung und der Vorstand als grundlegende Vereinsorgane benannt werden.

Die Mitgliederversammlung als das oberste Organ des Vereins wird definiert, indem

- die Bedingungen ihrer Berufung bestimmt werden,

- die Bedingungen ihrer Beschlussfähigkeit beschrieben werden,

- die Bedingungen der Beschlussfassung festgelegt werden,

- ihre Kompetenzen und Aufgaben aufgelistet werden,

- die Form der Beurkundung ihrer Beschlüsse genannt wird.

Der Vorstand wird als Vereinsvertretung definiert, indem

- die Struktur des Vorstandes beschrieben wird,

- der Bestellungsmodus geregelt wird,

- die Amtszeit festgelegt wird,

- seine Kompetenzen und Aufgaben aufgelistet werden,

- die Form der Vorstandssitzungen fest-
Die Zwecke und ihre Realisierungsmittel werden festgelegt, indem

- die strategischen und operativen Ziele aufgelistet werden,

- die konkreten Mittel der Zielrealisierung genannt werden.

Die Mitgliedschaft wird geregelt, indem

- die Voraussetzungen der Mitgliedschaft bestimmt werden,

- die Bedingungen der Aufnahme, der Streichung sowie die Rechte und Pflichten der Mitglieder festgelegt werden.

Die Organisationsstruktur des Vereins wird definiert, indem

- die Mitgliederversammlung, der Vorstand und die Kontroll-/Prüfkommission als grundlegende Vereinsorgane benannt werden.

Die Mitgliederversammlung als das oberste Organ des Vereins wird definiert, indem

- die Bedingungen ihrer Berufung bestimmt werden,

- die Bedingungen ihrer Beschlussfähigkeit beschrieben werden,

- die Bedingungen der Beschlussfassung festgelegt werden,

- ihre Kompetenzen und Aufgaben aufgelistet werden.

Der Vorstand wird als Vereinsvertretung definiert, indem

- die Struktur des Vorstandes beschrieben wird,

- der Bestellungsmodus geregelt wird,

- die Amtszeit festgelegt wird,

- seine Kompetenzen und Aufgaben aufgelistet werden,

- die Form der Vorstandssitzungen festgelegt wird,

- die Bedingungen der Beschlussfä- 


\begin{tabular}{|c|c|}
\hline $\begin{array}{l}\text { gelegt wird, } \\
\text { - die Bedingungen der Beschlussfä- } \\
\text { higkeit und der Beschlussfassung be- } \\
\text { stimmt werden, } \\
\text { - die Form der Beurkundung seiner Be- } \\
\text { schlüsse genannt wird. } \\
\text { Die Satzungsänderung und Vereins- } \\
\text { auflösung wird definiert, indem } \\
\text { - die Bedingungen (wer und wann) der } \\
\text { Satzungsänderung und der Vereins- } \\
\text { auflösung festgelegt werden. }\end{array}$ & $\begin{array}{l}\text { higkeit und der Beschlussfassung } \\
\text { bestimmt werden. } \\
\text { Die Revisionskommission wird als } \\
\text { Kontrollorgan definiert, indem } \\
\text { - die Struktur der Kommission be- } \\
\text { schrieben wird, } \\
\text { - der Bestellungsmodus geregelt wird, } \\
\text { - die Amtsperiode festgelegt wird, } \\
\text { - ihre Kompetenzen und Aufgaben } \\
\text { aufgelistet werden. } \\
\text { Das Vereinsvermögen wird festgelegt, } \\
\text { indem } \\
\text { - die Quellen der Finanzierung genannt } \\
\text { werden, } \\
\text { - die Bedingungen der Kooperationen } \\
\text { beschrieben werden. } \\
\text { Die Satzungsänderung und Ver- } \\
\text { einsauflösung wird definiert, indem } \\
\text { - die Bedingungen (wer und wann) der } \\
\text { Satzungsänderung und der Ver- } \\
\text { einsauflösung festgelegt werden. }\end{array}$ \\
\hline
\end{tabular}

Auf den ersten Blick fällt die Komplexität der polnischen Variante auf, die im Vergleich zur deutschen um einige Elemente reicher ist. Die Analyse des präskriptiven als auch des deskriptiven Textmusters macht deutlich, dass sich diese Textmuster voneinander unterscheiden. Das polnische Recht führt das Instrument der finanziellen Kontrolle in Form der Revisionskommission und einer Regelung bezüglich des Vereinsvermögens ein, während die deutschen Satzungen die Beurkundung der Beschlüsse vorschreiben.

Im Folgenden soll exemplarisch an einer Teilhandlung gezeigt werden, wie diese in beiden Sprachen sprachlich realisiert wird. Dabei soll geprüft werden, mit welchen Ausdrucksmitteln die in den exemplarischen Teilhandlungen enthaltenen Rechtsnormen, die in Verboten, Geboten und Erlaubnissen zum Ausdruck kommen, realisiert werden. SAYATZ (1996:278) spricht in diesem Zusammenhang von der deontischen Modalität, mit der sie die Beziehung zwischen Sachverhalten in Bezug auf „mögliche Welten“ relativ zu einem deontischen Redehintergrund (einer genauen bestimmten Rechtsordnung) beschreibt. Anders ausgedrückt: Die deontische Modalität legt die Möglichkeit oder die Notwendigkeit einer Handlung fest und wird daher als Modalität der Normen, der Gebote und Verbote bezeichnet. Und da deontische Sachverhalte 
sich als Möglichkeits- oder Notwendigkeitssachverhalte erfassen lassen, werden zur Beschreibung der deontischen Modalität im Deutschen neben den Modalverben wie können, müssen, dürfen und sollen auch ihre Ersatzformen wie verpflichtet sein, berechtigt sein, befugt sein, erforderlich sein, das Recht haben, verantwortlich sein, (un)zulässig sein, bedürfen/brauchen sowie modale Infinitivkonstruktionen mit haben $z u$ und sein $z u$ verwendet. ${ }^{5}$ Das Polnische bedient sich ebenfalls der Modalverben (wie móc, musieć, być powinnym) sowie modalisierter Konstruktionen (wie mieć obowiazek, prawo, być zobowiazanym), die semantisch und syntaktisch die Modalverben ersetzen. In beiden Sprachen werden zum einen passivisch gebrauchte Vollverben verwendet und zum anderen Konstruktionen, die eine Kombination von Verben mit Substantiven und Adjektiven oder Präsens-Partizipien darstellen. Darüber hinaus spielt auch die Verneinung eine wichtige Rolle, die zur Umkehrung der Modalität beiträgt, wenn etwa eine durch dürfen ausgedrückte Erlaubnis zu einem Verbot wird: man darf nicht, keiner darf (BRANDT 1996:233). Im Weiteren soll die deutsche Version der Regelung über die Mitgliederversammlung vorgestellt werden, und zwar am Beispiel der Satzung des MitOst e.V., Verein für Sprach- und Kulturaustausch in Mittel-, Ost- und Südosteuropa.

$\S 16$ Mitgliederversammlung

(1) In der Mitgliederversammlung hat jedes Mitglied - auch ein Ehrenmitglied eine Stimme. Die Übertragung der Ausübung des Stimmrechts auf andere Mitglieder ist nicht zulässig.

(2) Die Mitgliederversammlung ist für folgende Angelegenheiten zuständig:

1) Wahl, Abberufung und Entlastung des Vorstandes nach Vorlage des Kassenberichts,

2) Beschlussfassung über Änderungen der Satzung und über die Vereinsauflösung,

3) Ernennung von Ehrenmitgliedern,

4) weitere Aufgaben, soweit sich dies aus der Satzung oder nach dem Gesetz ergibt.

(3) Die ordentliche Mitgliederversammlung findet jährlich möglichst im ersten Quartal statt. Sie wird vom Vorstand mit einer Frist von sechs Wochen unter Angabe der Tagesordnung durch schriftliche Einladung einberufen.

(4) Die Tagesordnung ist zu ergänzen, wenn dies ein Mitglied bis spätestens eine Woche vor dem angesetzten Termin schriftlich fordert. Die Ergänzung ist zu Beginn der Versammlung bekanntzumachen.

$5 \quad$ Mehr zu den sprachlichen Ausdrucksformen deontischer Modalität bei SAYATZ (1996), VIEHWEGER / SPIES (1987) und BRANDT (1996). 
(5) Außerordentliche Mitgliederversammlungen sind auf Antrag der Mitglieder einzuberufen, wenn $1 / 3$ der Vereinsmitglieder die Einberufung schriftlich unter Angabe der Gründe verlangen.

(6) Die Mitgliederversammlung ist beschlussfähig, wenn sie ordnungsgemäß einberufen wurde.

(7) Beschlüsse der Mitgliederversammlung werden mit einfacher Mehrheit gefasst, Satzungsänderungen bedürfen einer 3/4-Mehrheit der anwesenden Mitglieder. Hierbei kommt es auf die abgegebenen gültigen Stimmen an.

(8) Die Mitgliederversammlung wählt einen Versammlungsleiter und einen Protokollführer.

(9) Über den Verlauf der Mitgliederversammlung ist ein Protokoll zu erstellen, das von dem Versammlungsleiter und dem Protokollführer zu unterzeichnen ist.

Schaut man sich die syntaktischen Konstruktionen an, so fällt zunächst auf, dass das kategorische Präsens (findet statt) verwendet wird. Nicht unüblich sind Passivkonstruktionen (werden gefasst). Die deontische Modalität wird meistens durch Modalkonstruktionen (ist zu ergänzen) und Modalausdrücke (nicht zulässig, bedürfen), nicht selten auch durch Konditionale (wenn dies ein Mitglied fordert) realisiert. Es handelt sich um das Konditionalgefüge, das jeweils eine Norm einbettet.

Als Beispiel für die entsprechende polnische Regelung der Mitgliederversammlung eines Vereins soll die Satzung des, Vereins der Kreativen Initiative „ę“", Towarzystwo Inicjatyw Twórczych „„e“ dienen.

Walne Zgromadzenie Członków

$\S 22$

Najwyższą władzą Stowarzyszenia jest Walne Zgromadzenie Członków.

(1) Walne Zgromadzenie jest zwoływane przez Zarząd jeden raz w roku lub częściej na pisemny i uzasadniony wniosek Komisji Rewizyjnej lub połowy członków Stowarzyszenia z inicjatywy własnej.

(2) W każdym z przypadków opisanych w pkt. 1. Zarząd jest zobowiązany do bezwarunkowego zwołania Walnego Zgromadzenia.

(3) O miejscu, terminie i porządku obrad Zarząd powiadamia członków na 14 dni przed terminem Walnego Zgromadzenia Członków.

(4) W Walnym Zgromadzeniu winna uczestniczyć co najmniej połowa członków uprawnionych do głosowania w pierwszym terminie, a w drugim terminie, który może być wyznaczony 30 minut później tego samego dnia jego uchwały są ważne bez względu na liczbę uczestników.

(5) W Walnym Zgromadzeniu mogą uczestniczyć członkowie zwyczajni Stowarzyszenia oraz, z głosem doradczym, członkowie wspierający i honorowi oraz zaproszeni goście. 
(6) Do kompetencji Walnego Zgromadzenia należy:

a. uchwalenie i zmiana regulaminu Walnego Zgromadzenia Członków

b. powoływanie i odwoływanie członków Zarządu i Komisji Rewizyjnej,

c. uchwalenie zmian statutu,

d. podejmowanie uchwały o rozwiązaniu Stowarzyszenia,

e. powoływanie członków honorowych

f. ustalanie wysokości składek członkowskich i wpisowego.

(7) Odwoływanie członków Zarządu, Komisji Rewizyjnej, rozwiązanie Stowarzyszenia wymaga większości $2 / 3$ głosów przy obecności przynajmniej połowy członków Stowarzyszenia.

Auch im Polnischen werden das kategorische Präsens (zarzqd powiadamia) sowie das Passiv (jest zwotywany) verwendet. Die deontische Modalität wird realisiert durch Konstruktionen wie jest zobowiazany, winna uczestniczyć, może być, wymaga, die als Ausdruck der Möglichkeit und Notwendigkeit verwendet werden. Obige Beispiele zeigen, dass die Mehrheit der Sätze im Indikativ formuliert ist, sowohl im Aktiv als auch im Passiv. Dass das Passiv im Polnischen weniger gebraucht wird, hat seine Ursachen in einer allgemeinsprachlichen Tendenz. Es soll nun anhand von Beispielen aus zehn Satzungstexten (jeweils fünf aus jeder Sprache) geprüft werden, in welchem qualitativen Verhältnis sprachlich markierte modalisierte Äußerungen zu formal nicht modalisierten stehen und welche Mittel zum Ausdruck von Geboten, Verboten und Erlaubnissen in beiden Sprachen verwendet werden. Diese Analyse hat zum Ziel, das Verhältnis zwischen Handlungsobligationen (Geboten und Verboten) und Handlungsoptionen (Erlaubnissen) in den Vereinssatzungen zu untersuchen. Die Handlungsobligationen werden durch sprachliche Mittel ausgedrückt, die die Notwendigkeit einer Handlung ausdrücken bzw. suggerieren. In deutschen Satzungstexten stellt sich das folgendermaßen dar. Zum Ausdruck der deontischen Notwendigkeit werden im Deutschen am häufigsten die Konstruktion ist...zu, die Konstruktion hat...zu, das verneinte Modalverb dürfen, die Modalverben müssen und sollen verwendet.

- Die Beitrittserklärung ist schriftlich vorzulegen.

- $\quad$ Einladungen zur Mitgliederversammlung haben durch den Vorstand vier Wochen vor der Versammlung zu erfolgen.

- $\quad$ Es darf keine Person durch Ausgaben, die dem Zweck der Körperschaft fremd sind, oder durch unverhältnismäßig hohe Vergütungen begünstigt werden. 
- In der Mahnung muss auf die bevorstehende Streichung der Mitgliedschaft hingewiesen werden.

- $\quad[. .$.$] dass die Vertretung durch den Vorsitzenden und einen stellvertreten-$ den Vorsitzenden oder im Verhinderungsfall des Vorsitzenden von den beiden stellvertretenden Vorsitzenden erfolgen soll.

Die Modalverben werden häufig auch in einer Passivkonstruktion verwendet, um die deontische Notwendigkeit abzuschwächen. Darüber hinaus werden auch die bereits oben erwähnten Konstruktionen verwendet, die eine deontische Notwendigkeit ausdrücken: ist verpflichtet, die Pflicht haben, bedürfen/brauchen, erforderlich und nicht zulässig sein.

- $\quad$ Bei Ablehnung des Antrags ist er nicht verpflichtet, der oder dem Antragstellenden die Gründe mitzuteilen.

- Die Mitglieder haben die Pflicht, semesterweise einen Mitgliedsbeitrag zu entrichten.

- $\quad$ Beschlüsse der Mitgliederversammlung werden mit einfacher Mehrheit gefasst, Satzungsänderungen bedürfen einer $3 / 4-$ Mehrheit der anwesenden Mitglieder.

- $\quad$ Die Übertragung der Ausübung des Stimmrechts auf andere Mitglieder ist nicht zulässig.

- Zur Änderung der Satzung sowie zur Auflösung des Vereins ist jedoch eine Mehrheit von drei Vierteln der abgegebenen gültigen Stimmen erforderlich.

Im Polnischen hingegen werden zum Ausdruck der deontischen Notwendigkeit vor allem folgende Konstruktionen verwendet: jest zobowiazany, nie może, jest wymagany, wymagać, powinno się, winno, należy, musieć, jest konieczny.

- Osoby nieobecne na Walnym Zebraniu zobowiazane sq złożyć pisemna zgodę na członkostwo w Komisjach Kwalifikacyjnych.

- $\quad$ Członek Zarzadu nie może być stałym członkiem Komisji Kwalifikacyjnej.

- Dla ważności innych pism i dokumentów wymagane sq dwa podpisy: prezesa lub wiceprezesa oraz członka Zarzadu.

- Uchwalenie Statutu lub jego zmiany oraz podjęcie uchwaty o rozwiazaniu Stowarzyszenia przez Walne Zebranie Członków wymaga kwalifikowanej większości głosów (2/3) przy obecności co najmniej połowy uprawnionych do głosowania. 
- Uchwała o rozwiazaniu stowarzyszenia powinna określać sposób likwidacji i przeznaczenie majatku zlikwidowanego stowarzyszenia.

- Walnym Zebraniu oraz o jego proponowanym porzadku obrad członkowie winni być zawiadomieni pisemnie co najmniej dwa tygodnie przed jego terminem.

- Jeśli rada uchwali swój regulamin, należy go przedtożyć zarzqdowi.

- Dwaj cztonkowie zarzqdu, z których przynajmniej jeden musi być przewodniczqcym lub zastępca przewodniczqcego, reprezentuja stowarzyszenie na zewnatrz.

- Kuratorium samodzielnie uchwala swój regulamin i obraduje wówczas, gdy uważa to za konieczne.

Die Handlungsoptionen werden mithilfe des Modalverbs können sowie der Konstruktionen berechtigt sein, zulässig sein und das Recht haben realisiert.

- Die Mitgliederversammlung ist berechtigt, weitere Organe zu bilden.

- Zulässig sind Erstattungen für notwendig entstandene Auslagen, die der Vereinsarbeit zugute kommen.

- Die Mitglieder haben das Recht, an den Mitgliederversammlungen des Vereins teilzunehmen.

Die deontische Möglichkeit wird im Polnischen durch das Modalverb móc und mieć prawo ausgedrückt.

- Stowarzyszenie może być członkiem krajowych i międzynarodowych organizacji o tym samym lub podobnym profilu działalności.

- Od negatywnej uchwaty zarzqdu przystuguje zainteresowanemu prawo wniesienia odwołania do zebrania cztonków, $w$ terminie 14 dni od doręczenia uchwaty.

Vergleicht man die sprachlichen Realisierungen der Vereinssatzungen, so ist zunächst festzustellen, dass in den deutschen Satzungstexten häufiger modalisierte bzw. deontische Ausdrücke $(0,30 \%$ aller Ausdrucksmittel) verwendet werden als in den polnischen Satzungen (0,20\% aller Ausdrucksmittel). Auch unter dem Aspekt der Handlungsobligation und Handlungsoption weisen die beiden Sprachen einen Unterschied auf. In den polnischen Satzungstexten dominieren vorwiegend Mittel zum Ausdruck von Handlungsoptionen mit $0,012 \%$ gegenüber $0,008 \%$ der Ausdrücke von Handlungsnotwendigkeiten, während in den deutschen Satzungen die Handlungsobligationen mit 0,020 \% 
gegenüber 0,014 \% der Ausdrücke von Möglichkeiten überwiegen. In diesem Zusammenhang könnte man auf die von BRANDT (1996) aufgestellte These verweisen: je demokratischer der Staat, desto mehr Optionen in den Gesetzen. Sie ist eine nachvollziehbare Erklärung für die Entwicklung der Rechtssprache im 18. und 19. Jahrhundert, aber schon für das 20. Jahrhundert hat sie ihre Gültigkeit verloren. Auch im interkulturellen bzw. kontrastiven Vergleich ist diese Erklärung eher gefährlich, da sie dem einen oder anderen demokratischen Staat wenig Demokratie unterstellen könnte. Zwar ist Polen im Vergleich zu Deutschland ein Land mit einer ,jüngeren“ Demokratie und mit einer unterbrochenen bürgerlichen Vereinstradition, folglich müssten dann jedoch in den polnischen Satzungstexten häufiger Handlungsobligationen zu finden sein als in den deutschen Satzungen.

\section{Zusammenfassung}

Vergleicht man deutsche und polnische Vereinssatzungen auf der textuellen Ebene, so ist zunächst festzuhalten, dass trotz einer großen Ähnlichkeit der Textkonstituenten einige Unterschiede bestehen, die sich mit Blick auf die jeweilige Vereinstradition erklären lassen. Wie eingangs festgestellt, werden die Vereine in Deutschland eher als Partner der öffentlichen Verwaltung und des Staates aufgefasst. Dabei ist wichtig, dass zwischen den Partnern ein regulärer Informationsaustausch stattfindet, der nach bestimmten Regeln erfolgen soll. Die Beurkundung der Vereinsbeschlüsse etwa ist ein Instrument, das den Informationsfluss nicht nur nach unten (gegenüber den Mitgliedern), sondern auch nach oben (gegenüber der öffentlichen Verwaltung) möglich macht. Sichtbar wird hier das Bestreben des Staates, für klare Kommunikationsstrukturen innerhalb des Vereins zu sorgen. Eine wichtige Rolle spielt in diesem Zusammenhang die Transparenz der Regel. Anders stellt sich der Sachverhalt in Polen dar, wo die Vereine als Auftragnehmer der öffentlichen Verwaltung verstanden werden, die einer Kontrolle bedürfen. Gemeint ist vor allem die Kontrolle im Bereich der Finanzierung und der Ausgaben der Vereine, die durch die Einrichtung der Revisionskommission und die Regelungen hinsichtlich der Finanzierungsquelle sichergestellt wird. Auch vor dem Hintergrund, dass junge Demokratien Gefahr laufen, sich nicht immer an klare Kommunikationsvereinbarungen zu halten, schärft diese Regelung das Bewusstsein für dieses Problem.

Vergleicht man die Vereinssatzungen im Polnischen und im Deutschen auf der sprachlichen Ebene, so ist festzustellen, dass die deutschen Satzungen eher Handlungsobligationen und die polnischen Satzungen Handlungsoptionen aus- 
drücken. Auch dieser Unterschied ist nur mit Bezug auf den jeweils kulturell bedingten Geltungsbereich der Textsorte erklärbar. Dem Vereinswesen werden in Polen immer noch Vorbehalte entgegengebracht. Das bürgerliche Engagement sowie der Zusammenschluss der Bürger werden noch lange nicht als eine Selbstverständlichkeit betrachtet. Da also in Bezug auf die zivilgesellschaftliche Entwicklung in Polen im Vergleich zu Deutschland noch vieles offen ist, müssen die Satzungen diese Tatsache auch widerspiegeln. Vor diesem Hintergrund werden in polnischen Satzungstexten meist Angebote und Vorschläge gemacht, die zwar konkrete Handlungsrahmen definieren, jedoch auch Raum für Ermutigung und Gestaltungsmöglichkeiten schaffen. Die deutschen Satzungen bestimmen durch Handlungsobligationen klare Handlungsregeln, da in Deutschland ein anderes Bewusstsein für das bürgerliche Engagement vorhanden ist. Auffallend ist in der Textsorte Satzung in beiden Sprachen, dass die textuelle Ebene eng mit den Regeln des Staates korrespondiert, während die sprachliche Ebene vorwiegend die Interessen der Zielgruppe berücksichtigt, also den kulturbedingten Bedarf der Vereinsmitglieder widerspiegelt. Erwähnung verdient noch die Tatsache, dass bei der Bestimmung der Handlungsobligationen und Handlungsoptionen nur auf die modalen Ausdrucksmittel zurückgegriffen wird, basierend auf der Annahme, dass nur sie Träger der Gebote, Verbote und Erlaubnisse in den Satzungen sein können. Weiter erforscht werden sollte, welcher anderen Mittel sich beide Sprachen bedienen, um die Normativität der Satzungen auszudrücken. Wichtig dabei erscheint die Untersuchung von performativen Operatorenbennenungen sowie von Nominalgruppen, die Normativität definieren.

\section{Literatur}

ADAMZIK, KIRSTEN (2001): Grundfragen einer kontrastiven Textologie. In: ADAMZIK, KIRSTEN (ed.): Kontrastive Textologie: Untersuchungen zur deutschen und französischen Sprach- und Literaturwissenschaft. Tübingen, 13-48.

BARTMIŃSKI, JERZY (1998): Tekst jako przedmiot tekstologii lingwistycznej. [Der Text als Gegenstand der linguistischen Textologie]. In: BONIECKA, BARBARA / BARTMIŃSKI, JERZY (eds.): Tekst. Problemy teoretyczne. Lublin, 9-25.

Bilut-Homplewicz, Zofia (2004): Textsortenspezifik als Desiderat in der deutschpolnischen und polnisch-deutschen kontrastiven Linguistik. In: HERWIG, ROLF (ed.): Sprache und die modernen Medien. Akten des 37. Linguistischen Kolloquiums in Jena 2002. Frankfurt (M.), 387-395.

Brandt, WolfGANG (1996): Handlungsobligationen und Handlungsoptionen. Modalverben und ihre verbalen Ersatzformen in der deutschen Gesetzessprache. In: HENNIG, 
JÖRG / MEIER, JÜRGEN (eds.): Varietäten der deutschen Sprache. Festschrift für Dieter Möhn. Frankfurt (M.), 229-246.

CZAChur, Waldemar (2005): Textsorten des Bereichs Vereinswesens. In: Studia Germanica Universitatis Vesprimiensis 2:111-126.

DiMTER, MATTHIAS (1981): Textklassenkonzepte heutiger Alltagssprache: Kommunikationssituation, Textfunktion und Textinhalt als Kategorien alltagssprachlicher Textklassifikation. Tübingen.

GAJDA, STANISŁAW (1993): Gatunkowe wzorce wypowiedzi. [Gattungsmuster der Äußerungen]. In: BARTMIŃSKI, JERZY (ed.): Encyklopedia kultury polskiej XX wieku. Bd. 2: Wspótczesny język polski. [Die polnische Sprache der Gegenwart]. Wrocław, 245-258.

GruCZA, SAMBOR (1993): Zur konfrontativen Textlinguistik. In: DARSKI, JÓZEF / Vetulani, Zygmunt (eds.): Sprache - Kommunikation - Informatik. Akten des 26. Linguistischen Kolloquiums Poznan 1991. Poznań, 425-429.

Gülich, Elsabeth / Raible, WolfGang (1975): Textsorten. Differenzierungskriterien aus linguistischer Sicht. Frankfurt (M.).

Hartmann, Peter (1971): Texte als linguistisches Objekt. In: STEMPEL, Wolf-Dieter (ed.): Beiträge zur Textlinguistik. München, 9-29.

Heinemann, Margot / Heinemann, WolfGang (2002): Grundlagen der Textlinguistik. Interaktion - Text - Diskurs. Tübingen.

HeINEMANn, WOLFGANG (2000): Textsorte, Textmuster, Texttyp. In: BRINKER, KLAUS et alii (eds.): Text- und Gesprächslinguistik. Ein internationales Handbuch zeitgenössischer Forschung. Berlin/New York, 507-523.

Heinemann, Wolfgang / Bilut-Homplewicz, Zofia (2005): Anmerkungen zur germanistischen Textlinguistik in Polen. In: Convivium:237-257.

Heinemann, Wolfgang / Viehweger, Dieter (1991): Textlinguistik. Eine Einführung. Tübingen.

HöRMANN, HANS (1977): Psychologie der Sprache. Berlin/Heidelberg.

IsENBERG, HoRST (1978): Probleme der Texttypologie. Variationen und Determination von Texttypen. In: Wissenschaftliche Zeitschriften der Karl-Marx-Universität Leipzig 27/5:565-579.

LERCHNER, GOTTHARD (1991): Zur empirischen Beschreibung von Textmustermischungen in einem historischen Textsortenspektrum. In: MACKELDEY, ROGER (ed.): Textsorten/Textmuster in der Sprech- und Schriftkommunikation. Festschrift zum 65. Geburtstag von Wolfgang Heinemann. Leipzig, 61-67.

LEŚ, EwA (2000): Od filantropii do pomocniczości. Studium porównawcze rozwoju $i$ działalności organizacji społecznych. [Von der Philanthropie zur Hilfeleistung. Vergleichende Studie zur Entwicklung und Tätigkeit gesellschaftlicher Organisationen]. Warszawa.

MaZur, Matgorzata (1997): Packungsbeilage als Textmuster. In: Lubelskie Materiaty Neofilologiczne 21:193-216. 
Michel, GeORG (1990): Textmuster und Stilmuster. In: BAHNER, WERnER / SCHILDT, JoAchim / Viehweger, DieTer (eds.): Proceedings of the Fourteenth International Congress of Linguists. Bd. 3. Berlin, 2178-2180.

MistRIK, JOZEF (1973): Exakte Typologie von Texten. München.

Riehl, Claudia M. (2001): Schreiben, Text und Mehrsprachigkeit. Zur Textproduktion in mehrsprachigen Gesellschaften am Beispiel der deutschsprachigen Minderheiten in Südtirol und Ostbelgien. Tübingen.

SACHSSE, CHRISTOPH (2002): Traditionslinien bürgerschaftlichen Engagements in Deutschland. In: Aus Politik und Zeitgeschichte 9:3-5.

SANDIG, BARBARA (1986): Stilistik der deutschen Sprache. Berlin/New York.

- (1997): Formulieren und Textmuster. Am Beispiel von Wissenschaftstexten. In: JAKOBS, EvA-MARIA (ed.): Schreiben in der Wissenschaft. Frankfurt (M.), 25-44.

SAYATZ, UlRIKE (1996): Modale Referenz in Gesetzen und Gesetzeskommentierungen. Ein textvergleichender Ansatz. In: MOTSCH, WOLFGANG (ed.): Ebenen der Textstruktur. Sprachliche und kommunikative Prinzipien. Tübingen, 275-300.

SCHMIDT, SIEGFRIED J. (1976): Texttheorie. Probleme einer Linguistik der sprachlichen Kommunikation. München.

SCHRÖDER, THOMAS (2003): Die Handlungsstruktur von Texten. Ein integrativer Beitrag zur Texttheorie. Tübingen.

STEGer, Hugo (1983): Über Textsorten und andere Textklassen. In: VORSTAND DER VEREINIGUNG DER DEUTSCHEN HOCHSCHULGERMANISTEN (ed.): Textsorten und literarische Gattungen. Dokumentation des Germanistentages in Hamburg 1979. Berlin, 25-67.

VIEHWEGER, DIETER / SPIES, GOTTFRIED (1987): Struktur illokutiver Handlungen in Anordnungstexten. In: Motsch, WolfGANG (ed.): Satz, Text, sprachliche Handlung. Berlin, 81-118.

WARNKE, INGO H. (2001): Intrakulturell versus Interkulturell - zur kulturellen Bedingtheit von Textmustern. In: Fix, Ulla / Habscheid, Stephan / KLein, JoseF (eds.): Zur Kulturspezifik von Textsorten. Tübingen, 241-254.

WAWRZYNIAK, ZDZISŁAW (1975): Konfrontative Textlinguistik und Fremdsprachenunterricht. In: SZULC, ALEKSANDER (ed.): Sprachwissenschaft und Fremdsprachenunterricht. Poznań, 53-58.

- (1989): Zum Vorverständnis einer konfrontativen Textlinguistik. In: Zeszyty Naukowe Uniwersytetu Jagiellońskiego. Prace Językoznawcze 94:195-201.

WeRLICH, EGON (1975): Typologie der Texte. Entwurf eines textlinguistischen Modells zur Grundlegung einer Textgrammatik. Heidelberg.

WitosZ, BożENA (2001): Gatunek - sporny (?) problem wspótczesnej refleksji tekstologicznej. [Die Gattung - ein umstrittenes (?) Problem der gegenwärtigen textlinguistischen Reflexion]. In: Teksty Drugie 5:67-85. 
Konfrontative Textlinguistik

WoJTAK, MARIA (1999): Modlitwa ustalona - podstawowe wyznaczniki gatunku. [Das Gebet - grundlegende Textkennzeichnungen]. In: ADAMOWSKI, JAN / NIEBRZEGOWSKA, StANiSŁaWA (eds.): W zwierciadle języka kultuy. Lublin, 187-196.

Wyrwas, Katarzyna (2002): Skarga jako gatunek mowy. [Die Klage als Redegattung]. Katowice.

ŻMigrodZKA, BożEnA (1997): Testament jako gatunek tekstu. [Das Testament als Textgattung]. Katowice. 